\title{
Cosmological Constant from the Entropy Balance Condition
}

\author{
Merab Gogberashvili (iD ${ }^{1,2}$ \\ ${ }^{1} J a v a k h i s h v i l i$ Tbilisi State University, 3 Chavchavadze Avenue, Tbilisi 0179, Georgia \\ ${ }^{2}$ Andronikashvili Institute of Physics, 6 Tamarashvili Street, Tbilisi 0177, Georgia \\ Correspondence should be addressed to Merab Gogberashvili; gogber@gmail.com
}

Received 4 August 2018; Accepted 16 September 2018; Published 26 November 2018

Guest Editor: Cynthia Keeler

Copyright @ 2018 Merab Gogberashvili. This is an open access article distributed under the Creative Commons Attribution License, which permits unrestricted use, distribution, and reproduction in any medium, provided the original work is properly cited. The publication of this article was funded by SCOAP ${ }^{3}$.

\begin{abstract}
In the action formalism variations of metric tensors usually are limited by the Hubble horizon. On the contrary, variations of quantum fields should be extended up to the event horizon, which is the real boundary of the spacetime. As a result the entanglement energy of quantum particles across the apparent horizon is missed in the cosmological equations written for the Hubble volume. We identify this missing boundary term with the dark energy density and express it (using the zero energy assumption for the finite universe) as the critical density multiplied by the ratio of the Hubble and event horizons radii.
\end{abstract}

Many authors consider so-called emergent theories in which gravity is not a fundamental field, but like thermodynamics or hydrodynamics is defined for the matter in bulk [1-7]. One such approach is the thermodynamic model of gravity [3-15], where spacetime emerges from the properties of the "universal" ensemble of quantum particles. In this approach the entropy, rather than the energy density, plays the crucial role.

Since in General Relativity horizons are unavoidable and horizons block information, entropy and temperature can be introduced for spacetime. One such boundary is the apparent horizon with the radius (for the spatially flat universe),

$$
R_{H}=\frac{1}{H} \approx 14.5 G l y,
$$

where $H \equiv \dot{a} / a$ is the Hubble parameter. For the Hubble volume,

$$
V_{H}=\frac{4}{3} \pi R_{H}^{3},
$$

having the surface area

$$
A_{H}=4 \pi R_{H}^{2},
$$

one can associate the temperature $[16,17]$,

$$
T=\frac{1}{2 \pi R_{H}},
$$

and an entropy $[18,19]$,

$$
S_{H}=\frac{A_{H}}{4 G},
$$

where $G$ is the Newton constant.

The concept of entropy is a powerful tool in thermodynamics, information theory, and quantum physics and allows us to study different aspects of physical systems using a similar mathematical framework. In quantum mechanics a measurement is considered as the interaction of three systems: the quantum object, memory (measurement device), and observer. Then the total entropy of the ensemble of all quantum particles, which is formed by the information, statistical (thermodynamic), and quantum (entanglement) components [20,21], can be assumed to be zero [15]. In this case the universe can always remain in pure state and only allow a unitary time-evolution, as it is suggested by von Neumann's model. Also the "universal" entropy remains zero at all stages of universe's evolution, while any subsystem (for example, the Hubble volume) has nonzero entropy.

In our previous papers it was demonstrated that "world ensemble" approach is compatible with the existing fieldtheoretical descriptions, as the relativistic $[11,12]$ and quantum [13] properties are emerging from its properties. Moreover, the model allows us to explain the hierarchy problem in particle physics by the fact that our underlying assumption 
that any gravitational interaction of two particles involves interactions with all particles of the world ensemble effectively weakens the observed strength of gravity by the factor proportional to the number of particles in the ensemble [14].

As it was noted in [15], the convenient physical parameter to measure information can be an action, which in most cases is an additive quantity like entropy, also contains positive and negative components, and exhibits the unique discrete value, the action quantum $\hbar$ [22]. Then the maximum entropy principle in thermodynamics [23-25] and the least action principle in field theory lead to the same formalism. The relation of the classical action of a physical system to the thermodynamic entropy translates the condition of entropy neutrality into the zero action principle: the sum of all components of the action for a physical system (including the boundary terms) is zero. One consequence of this principle for the whole universe is the zero-energy condition,

$$
\rho_{U}=0,
$$

i.e., the total density of all the forms of energy in the universe, $\rho_{U}$, at any moment of time should be zero. This mean that the universe can emerge without violation of the energy conservation, which appears to be preferable point of view in cosmology $[26,27]$.

In this paper we want to connect the entanglement energy of quantum particles across the apparent horizon, which is missed in the classical cosmological equations written for the Hubble volume, with the dark energy (DE), origin of which remains a mystery [28]. It is known that DE can be modelled by Einstein's cosmological constant, for which a typical fit to current observational data gives $\Lambda \approx 3 \times 10^{-122} \mathrm{sec}^{-2}$. However, in quantum field theory, the natural value of $\Lambda$ is of the order of unity. This discrepancy is one of the biggest challenges in modern cosmology and fundamental physics $[29,30]$.

Alternatives of the introduction of a cosmological constant are that DE arises from the evolution of dynamical fields of an unknown origin, or modifications of General Relativity. In order to distinguish between these hypotheses, a worldwide effort is ongoing to measure the effective equation of state and clustering properties of DE, using wide field cosmological surveys [31]. For reviews on the DE and theories, see [32-35] and references therein.

One promising approach for solving the DE puzzle is the Holographic DE model [36-39], which is based on the quantum zero-point energy predicted by an effective quantum field theory. The primary model of this kind, which as the IR cut-off uses the apparent horizon of the universe, $R_{H}$, has serious drawbacks [40-43], since for the DE density predicts the value:

$$
\rho_{D E}=2 \pi \rho_{c}
$$

where

$$
\rho_{c}=\frac{3 H^{2}}{8 \pi G}
$$

denotes the critical density. As we see $\rho_{D E}$ in Holographic DE model (7) is larger than observed; in fact it even exceeds $\rho_{c}$. To solve this failure physicists are considering [44] (i) interactions between the cosmos sectors, (ii) various models for entropies, and (iii) different from $R_{H}$ cut-offs.

We note that, based on spacetime thermodynamics, a proper causal boundary of the classical spacetime is its apparent horizon $[45,46]$, meaning that the metric fluctuations are bounded by $R_{H}$ and also that thermodynamics laws are satisfied on this boundary [47, 48]. Moreover, the event horizon in the context of cosmology as well as in the context of a black hole is always defined globally, as the causal structure of spacetime is a global thing (see more discussions in [39]).

From the other hand, the quantum fluctuations of matter fields should be limited not by the Hubble horizon (1), but by the event horizon,

$$
R_{e}=\int_{1}^{\infty} \frac{d a}{a^{2} H(a)} \approx 16.7 G l y,
$$

which represents a real boundary of spacetime. Then the entanglement energy of quantum particles across the apparent horizon $R_{H}$, which is defined as disturbed vacuum energy due to the presence of a boundary [49], is missed in the cosmological equations written for the Hubble volume and can be taken into account by introduction of a boundary term. It was found that the perfect fluid of entanglement has a negative pressure [50] and can be interpreted as the origin of DE.

The terms corresponding to entanglement across $R_{H}$ should disappear in the equation of state of classical fields. Indeed, for the light-like geodesics, which describe the Hubble horizon in the spatially flat universe, the Einstein equations written in the form of the first law [4-8],

$$
\left(R_{\mu \nu}-\frac{1}{2} g_{\mu \nu} R\right) u^{\mu} u^{\nu}=8 \pi G T_{\mu \nu} u^{\mu} u^{\nu}
$$

do not contain the DE terms. This equation involves additional vector field $u^{\nu}$, but contains all information content of the ordinary tensorial Einstein equations, because it is demanded that it holds for all $u^{v}$. In addition, if one assumes that $u^{v}$ is an orthogonal to the observers horizon null vector field [5-8],

$$
g_{\mu \nu} u^{\mu} u^{\nu}=0
$$

in that obtained from (10) tensorial Einstein equations,

$$
R_{\mu \nu}-\frac{1}{2} g_{\mu \nu} R=8 \pi G\left(T_{\mu \nu}+g_{\mu \nu} \Lambda\right)
$$

$\Lambda$ arises as an integration constant, which is not connected with the large constant vacuum energy terms in matter Lagrangians and needs to be fixed using an extra physical principle. For example, since we know that information is a physical entity [51-55], it can be identified with the amount of information accessible to an eternal observer at the horizon [8], or with the energy of collective gravitational interactions of all particles in the finite universe $[9,10]$.

Equation (10) has the natural interpretation as the balance of gravitational and matter heat densities, where the right 
hand side represents the matter heat density, in the spirit of the first law of thermodynamics. This is obvious, for example, for the case of ideal fluid using the classical Gibbs-Duhem relation,

$$
T_{\mu \nu} u^{\mu} u^{\nu} \longrightarrow \rho+p=\frac{T S_{m}}{V}
$$

where $T$ is the temperature and $S_{m}$ is the entropy of the matter in the volume $V$.

Connections of the cosmological constant with the boundary conditions and generalized equations of state can be demonstrated also from the cosmological equations. For a homogeneous, isotropic, and flat universe $(k=0)$ there are two independent Friedmann equations with the cosmological $\operatorname{term} \Lambda$ :

$$
\begin{aligned}
H^{2} & =\frac{8 \pi G}{3} \rho+\frac{1}{3} \Lambda \\
\dot{H}+H^{2} & \equiv \frac{\ddot{a}}{a}=-\frac{4 \pi G}{3}(\rho+3 p)+\frac{1}{3} \Lambda .
\end{aligned}
$$

Using the first equation of (14) the second equation can be reexpressed without $\Lambda$,

$$
\dot{H}=-4 \pi G(\rho+p) \text {. }
$$

From system (14) one can obtain also the matter energymomentum conservation condition,

$$
\partial_{\nu} T^{\nu \mu}=0
$$

which leads to

$$
\dot{\rho}=-3 H(\rho+p)
$$

Due to the presence of derivatives in (16) the cosmological constant $\Lambda$ does not appear in (17) as well.

If instead of (14) one will choose (15) and (17) as the independent system of cosmological equations, $\Lambda$ obtains the role of integration constant which can be fixed from an equation of state. Indeed, combining (15) with (17) and integrating over the time we have

$$
H^{2}=\frac{8 \pi G}{3} \rho+C
$$

where the cosmological term reappears in the form of an arbitrary constant $C$, which should be chosen as

$$
C=\frac{1}{3} \Lambda
$$

in order to obtain the first Friedmann equation in (14).

To find the value of DE density in our approach let us estimate the entropy input in a region as the sum of the entropy flux (entropy received per unit surface) transferred through the boundary and the entropy supplied by internal sources (entropy generated per unit volume). If we neglect the entropy supplied by internal sources, then according to the Second Law of Thermodynamics the time derivative of the entropy contained within the volume, $S$, is equal to the flux of the matter entropy, $S_{m}$, through the boundary $A$,

$$
\frac{d S}{d t}=S_{m} A .
$$

Using the relations of types (5) and (13), (20) takes the form

$$
\frac{1}{4 G} \frac{d A}{d t}=\frac{\rho+p}{T} A
$$

For the Hubble volume, taking into account (3) and (4), this equation leads to one of the cosmological equations (15). Then combined with the energy conservation equation (17) and integrating over time we find again (18), where $C$ corresponds to some hidden amount of energy.

For the finite universe limited by the event horizon, $R_{e}$, the energy balance condition (18) should obtain the form

$$
\frac{1}{R_{e}^{2}}=\frac{8 \pi G}{3} \rho_{U},
$$

without the boundary term. Then the zero energy condition (6) allows us to fix $C$ in (18) as

$$
C=\frac{1}{R_{e}^{2}} .
$$

Therefore, using (1) and (9) for the DE density, we find

$$
\rho_{D E}=\rho_{c} \frac{C}{H^{2}}=\rho_{c} \frac{R_{H}^{2}}{R_{e}^{2}}=0.75 \rho_{c},
$$

which is very close to the observed value of the DE.

Note that the relation (24) must be valid at any time of cosmological evolution, which gives time-dependence of DE. Moreover, the parameter $C$ in the energy balance condition (18) can change the sign in the situation when the Hubble horizon crosses the event horizon. In General Relativity, as we know, $\Lambda$ is a true constant. However, an expanding universe is not expected to have a static vacuum energy density and there is the possibility that $\Lambda$ is actually a time dependent quantity. In order to implement the notion of a smoothly evolving vacuum energy density is not necessary to introduce ad hoc scalar fields [56-58], as usually done in quintessence formulations. Let us mention a dynamical approach based on extending the variational principle by promoting $\Lambda$ from being a parameter to a field [59-61]. In this modified variational approach the resulting history is indistinguishable from General Relativity with a constant value of $\Lambda$ put in by hand at the right value at each observational time. In our model $\Lambda$ also appears to be time dependent and even can be negative, positive, or zero at different stages of the universe evolution. This fact can be used to model, for example, the end of the inflation epoch.

To conclude, in this paper we estimate the DE density within the thermodynamic model of gravity using the zero energy condition: the total energy of the universe inside its event horizon is zero. We notice that in the action formalism variations of metric tensor should be limited by the Hubble horizon, which represents a causal boundary of 
classical spacetime. On the contrary, variations of quantum fields are limited by the event horizon, which is the real boundary of the spacetime. Then the entanglement energy of quantum particles across the apparent horizon is missed in the cosmological equations written for the Hubble volume. We identify this entanglement density with the DE, which can be introduced as a boundary term in the cosmological equations. In our model the DE density equals the critical density reduced by the ratio of the squares of the Hubble and event horizons radii (24), having the value in good agreement with the observational data. In our approach DE density appears to be time dependent and can change the sign, what can explain transitions form accelerations to decelerations at different epoches of cosmological evolution.

\section{Data Availability}

The data used to support the findings of this study are available from the corresponding author upon request.

\section{Conflicts of Interest}

The author declares that there are no conflicts of interest.

\section{References}

[1] A. Connes and C. Rovelli, "von Neumann algebra automorphisms and time-thermodynamics relation in generally covariant quantum theories," Classical and Quantum Gravity, vol. 11, no. 12, article 2899, 1994.

[2] C. Rovelli and M. Smerlak, "Thermal time and TolmanEhrenfest effect: 'temperature as the speed of time"' Classical and Quantum Gravity, vol. 28, no. 7, Article ID 075007, 2012.

[3] E. P. Verlinde, "On the origin of gravity and the laws of Newton," Journal of High Energy Physics, vol. 2011, no. 4, article 29, 2011.

[4] T. Jacobson, "Thermodynamics of spacetime: the Einstein equation of state," Physical Review Letters, vol. 75, no. 7, article 1260, 1995.

[5] T. Padmanabhan, "Thermodynamical aspects of gravity: new insights," Reports on Progress in Physics, vol. 73, no. 4, Article ID 046901, 2010.

[6] T. Padmanabhan, "Emergent Gravity Paradigm: Recent Progress," Modern Physics Letters A, vol. 30, no. 03n04, Article ID 1540007, 2015.

[7] T. Padmanabhan, "Distribution function of the atoms of spacetime and the nature of gravity," Entropy, vol. 17, no. 11, pp. 74207452, 2015.

[8] M. Gogberashvili and U. Chutkerashvili, "Cosmological constant in the thermodynamic models of gravity," Theoretical Physics, vol. 2, no. 4, pp. 163-165, 2017.

[9] M. Gogberashvili and I. Kanatchikov, "Cosmological parameters from the thermodynamic model of gravity," International Journal of Theoretical Physics, vol. 53, no. 5, pp. 1779-1783, 2014.

[10] M. Gogberashvili, "On the dynamics of the ensemble of particles in the thermodynamic model of gravity," Journal of Modern Physics, vol. 5, pp. 1945-1957, 2014.

[11] M. Gogberashvili, "Universal' FitzGerald contractions," The European Physical Journal C, vol. 63, no. 2, pp. 317-322, 2009.
[12] M. Gogberashvili and I. Kanatchikov, "Machian origin of the entropic gravity and cosmic acceleration," International Journal of Theoretical Physics, vol. 51, no. 3, pp. 985-997, 2012.

[13] M. Gogberashvili, "Thermodynamic gravity and the schrodinger equation," International Journal of Theoretical Physics, vol. 50, no. 8, pp. 2391-2402, 2011.

[14] M. Gogberashvili, "A machian solution of the hierarchy problem," The European Physical Journal C, vol. 54, no. 4, pp. 671674, 2008.

[15] M. Gogberashvili, "Information-probabilistic description of the universe," International Journal of Theoretical Physics, vol. 55, no. 9, pp. 4185-4195, 2016.

[16] S. W. Hawking, "Particle creation by black holes," Communications in Mathematical Physics, vol. 43, no. 3, pp. 199-220, 1975.

[17] S. W. Hawking, "Black holes and thermodynamics," Physical Review D: Particles, Fields, Gravitation and Cosmology, vol. 13, no. 2, article 191, 1976.

[18] J. D. Bekenstein, "Black holes and entropy", Physical Review D: Particles, Fields, Gravitation and Cosmology, vol. 7, no. 8, article 2333, 1973.

[19] J. D. Bekenstein, "Generalized second law of thermodynamics in black-hole physics," Physical Review D: Particles, Fields, Gravitation and Cosmology, vol. 9, no. 12, article 3292, 1974.

[20] W.-Y. Hwang, "A coherent view on entropy," Natural Science, vol. 6, no. 7, pp. 540-544, 2014.

[21] D. Song, "Negative entropy and black hole information," International Journal of Theoretical Physics, vol. 53, no. 4, pp. 13691374, 2014.

[22] A. Annila, "All in action," Entropy, vol. 12, no. 11, pp. 2333-2358, 2010.

[23] E. T. Jaynes, "Information theory and statistical mechanics," Physical Review Journals Archive, vol. 106, no. 4, article 620, 1957.

[24] E. T. Jaynes, “Gibbs vs Boltzmann entropies," American Journal of Physics, vol. 33, article 391, 1965.

[25] E. T. Jaynes, Maximum Entropy and Bayesian Methods in Inverse Problems, C. R. Smith and W. T. Grandy Jr., Eds., Reidel, Dordrecht, Germany, 1985.

[26] R. P. Feynman, F. B. Morinigo, and G. Wagner, Feynman Lectures on Gravitation, Addison-Wesley, Reading, MA, USA, 1995.

[27] S. Hawking, A Brief History of Time, Bantam, Toronto, Canada, 1988.

[28] M. Roos, Introduction to Cosmology, John Wiley and Sons, UK, 2003.

[29] S. Weinberg, "The cosmological constant problem," Reviews of Modern Physics, vol. 61, no. 1, article 1, 1989.

[30] M. P. Hobson, G. P. Efstathiou, and A. N. Lasenby, General Reltivity: An Introduction for Physicists, Cambridge University Press, Cambridge, UK, 2006.

[31] K. Bamba, S. Capozziello, S. Nojiri, and S. D. Odintsov, "Dark energy cosmology: the equivalent description via different theoretical models and cosmography tests," Astrophysics and Space Science, vol. 342, no. 1, pp. 155-228, 2012.

[32] T. Padmanabhan, "Cosmological constant-the weight of the vacuum," Physics Reports, vol. 380, no. 5-6, pp. 235-320, 2003.

[33] S. Capozziello and V. Faraoni, Beyond Einstein Gravity, Springer, Dordrecht, The Netherlands, 2010.

[34] A. Joyce, B. Jain, J. Khoury, and M. Trodden, "Beyond the cosmological standard model," Physics Reports, vol. 568, pp. 1-98, 2015. 
[35] S. Nojiri, S. D. Odintsov, and V. K. Oikonomou, "Modified gravity theories on a nutshell: Inflation, bounce and late-time evolution," Physics Reports, vol. 692, pp. 1-104, 2017.

[36] A. G. Cohen, D. B. Kaplan, and A. E. Nelson, "Effective field theory, black holes, and the cosmological constant," Physical Review Letters, vol. 82, no. 25, Article ID 4971, 1999.

[37] S. Thomas, "Holography stabilizes the vacuum energy," Physical Review Letters, vol. 89, no. 8, Article ID 081301, 2002.

[38] S. D. H. Hsu, "Entropy bounds and dark energy," Physics Letters B, vol. 594, no. 1-2, pp. 13-16, 2004.

[39] M. Li, "A model of holographic dark energy," Physics Letters B, vol. 603, no. 1-2, pp. 1-5, 2004.

[40] B. Guberina, R. Horvat, and H. Nikolić, "Non-saturated holographic dark energy," Journal of Cosmology and Astroparticle Physics, vol. 2007, no. 01, article 012, 2007.

[41] R. Horvat, "On the internal consistency of holographic dark energy models," Journal of Cosmology and Astroparticle Physics, vol. 2008, no. 10, article 022, 2008.

[42] S. Gao, "Explaining holographic dark energy," Galaxies, vol. 1, no. 3, pp. 180-191, 2013.

[43] S. Gao, "A conjecture on the origin of dark energy," Chinese Physics Letters, vol. 22, no. 3, article 783, 2005.

[44] S. Wang, Y. Wang, and M. Li, "Holographic dark energy," Physics Reports, vol. 696, pp. 1-57, 2017.

[45] S. A. Hayward, S. Mukhoyama, and M. C. Ashworth, "Dynamic black-hole entropy," Physics Letters A, vol. 256, no. 5-6, pp. 347350, 1999.

[46] D. Bak and S.-J. Rey, "Cosmic holography," Classical and Quantum Gravity, vol. 17, no. 15, article 83, 2000.

[47] R. G. Cai and S. P. Kim, "First law of thermodynamics and Friedmann equations of Friedmann-Robertson-Walker universe," Journal of High Energy Physics, vol. 2005, no. 02, article 050, 2005.

[48] M. Akbar and R. G. Cai, "Thermodynamic behavior of the Friedmann equation at the apparent horizon of the FRW universe," Physical Review D: Particles, Fields, Gravitation and Cosmology, vol. 75, Article ID 084003, 2007.

[49] S. Mukohyama, M. Seriu, and H. Kodama, "Can the entanglement entropy be the origin of black-hole entropy?" Physical Review D: Particles, Fields, Gravitation and Cosmology, vol. 55, no. 12, Article ID 7666, 1997.

[50] J.-W. Lee, J. Lee, and H.-C. Kim, "Dark energy from vacuum entanglement," Journal of Cosmology and Astroparticle Physics, vol. 2007, no. 08, article 005, 2007.

[51] R. Landauer, "Irreversibility and heat generation in the computing process," IBM Journal of Research and Development, vol. 5, no. 3, pp. 183-191, 1961.

[52] R. Landauer, "Information is physical," in Proceedings of the Workshop on Physics and Computation (PhysComp '92), IEEE Computer Society Press, Los Alamitos, Calif, USA, 1993.

[53] L. Brillouin, "The negentropy principle of information," Journal of Applied Physics, vol. 24, no. 9, Article ID 1152, 1953.

[54] L. Brillouin, Science and Information Theory, Academic, New York, NY, USA, 1962.

[55] L. Brillouin, Scientific Uncertainty and Information, Academic, New York, NY, USA, 1964.

[56] J. Sola, "Vacuum energy and cosmological evolution," AIP Conference Proceedings, vol. 1606, no. 1, article 19, 2014.

[57] J. Sola, "Cosmological constant and vacuum energy: old and new ideas," Journal of Physics: Conference Series, vol. 453, no. 1, Article ID 012015, 2013.
[58] J. Sola, "Cosmologies with a time dependent vacuum," Journal of Physics: Conference Series, vol. 283, no. 1, Article ID 012033, 2011.

[59] D. J. Shaw and J. D. Barrow, "A testable solution of the cosmological constant and coincidence problems," Physical Review D: Particles, Fields, Gravitation and Cosmology, vol. 83, no. 4, Article ID 043518, 2011.

[60] J. D. Barrow and D. J. Shaw, "New solution of the cosmological constant problems," Physical Review Letters, vol. 106, no. 10, Article ID 101302, 2011.

[61] J. D. Barrow and D. J. Shaw, "The value of the cosmological constant," General Relativity and Quantum Cosmolog, vol. 43, no. 10, pp. 2555-2560, 2011. 

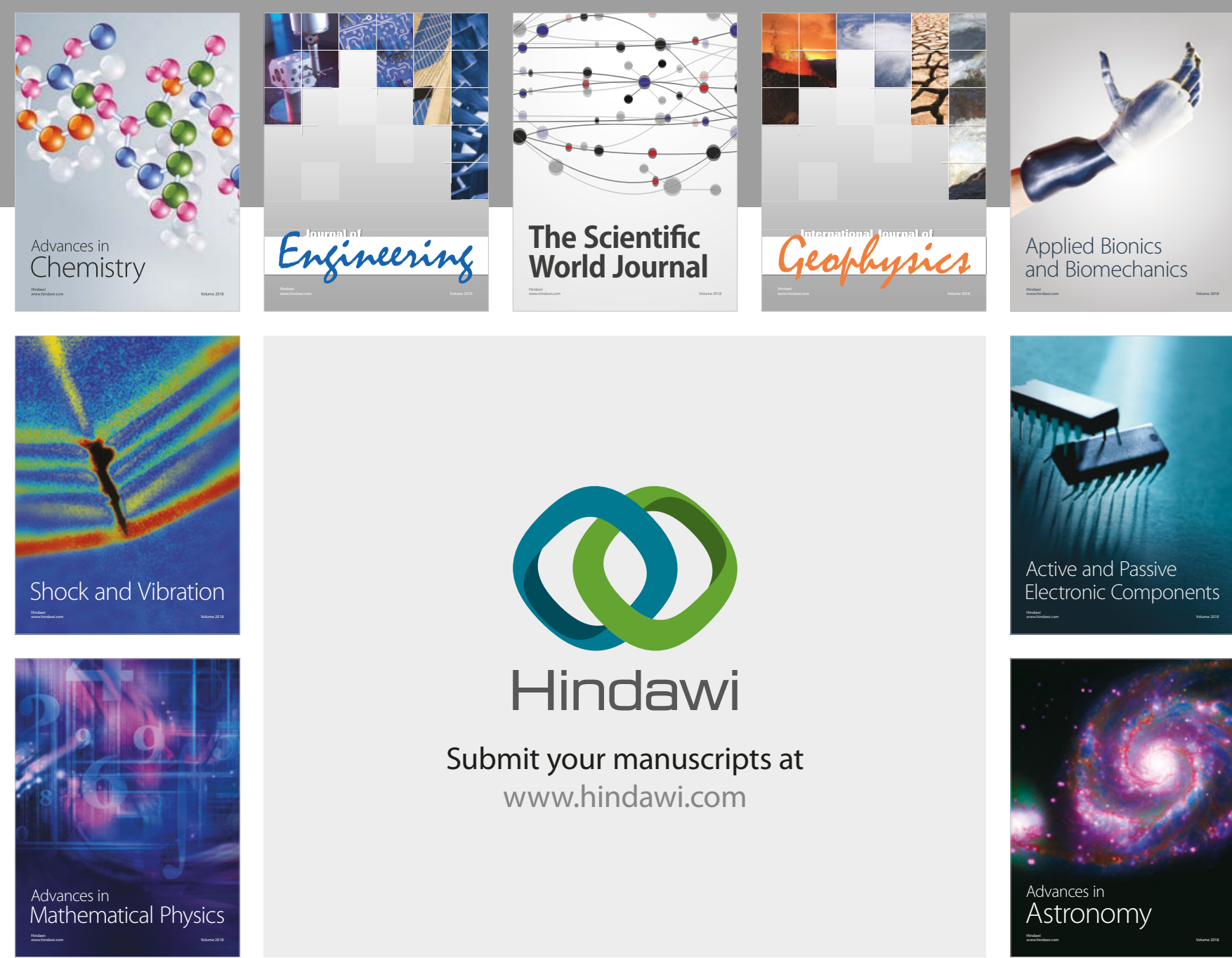

Submit your manuscripts at

www.hindawi.com

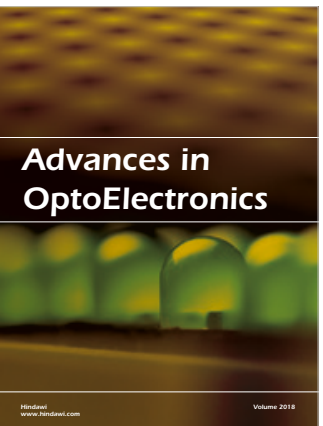

\section{Rotcting Machinery}
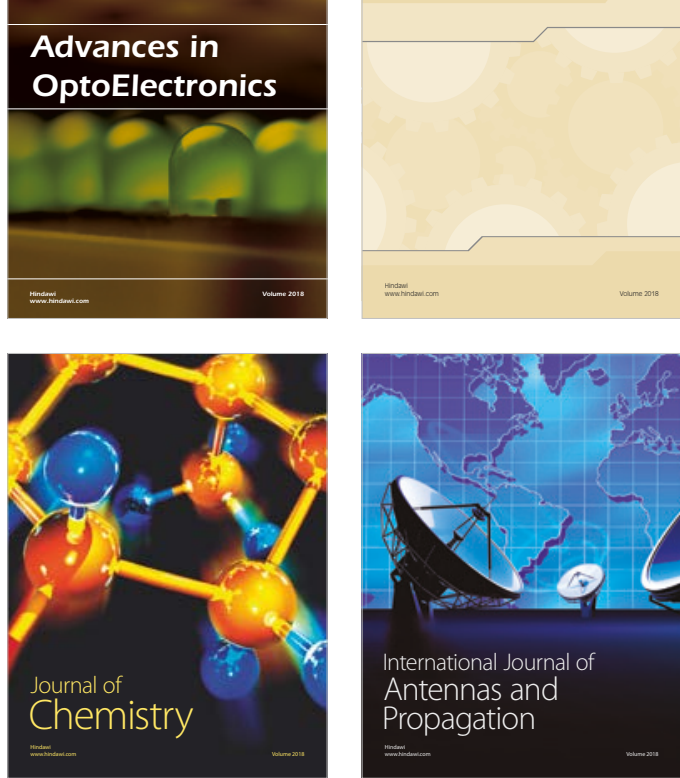

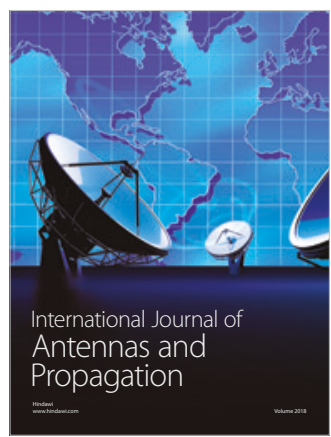

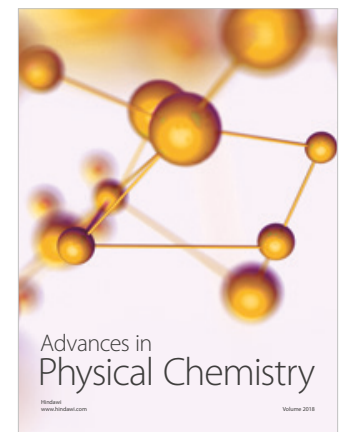

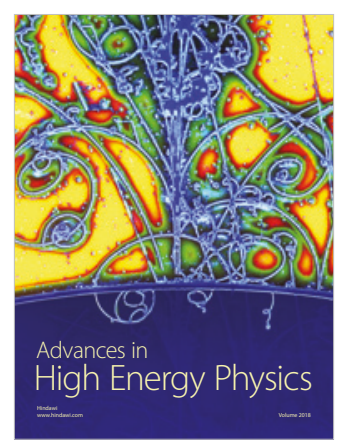

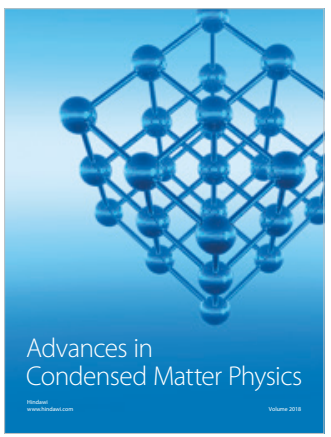

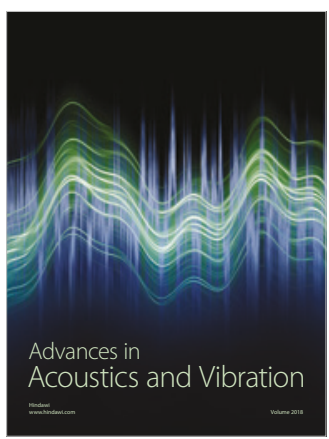

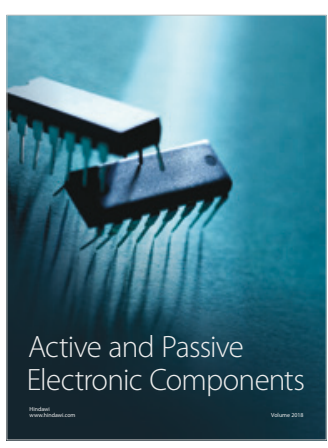
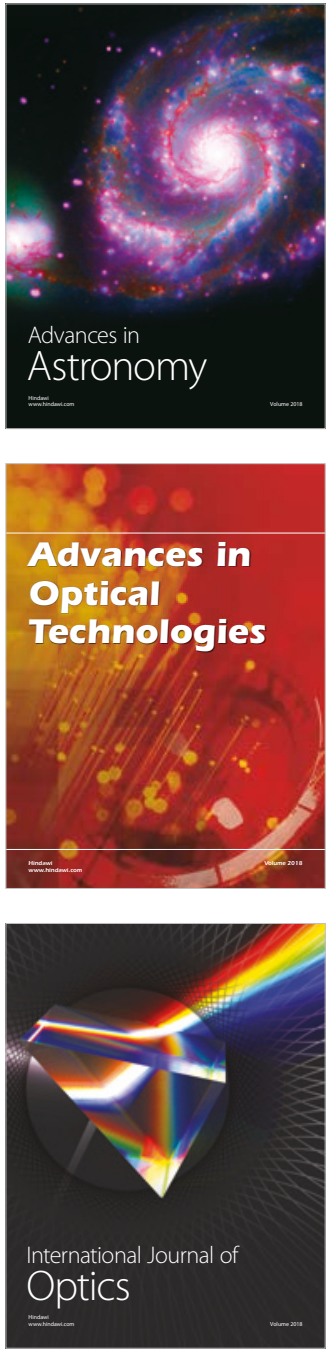